\section{Attachment, proliferation and differentiation of periodontal ligament cells on various guided tissue regeneration membranes}

\author{
Takata T, Wang H-L, and Miyauchi M: Attachment, proliferation and \\ differentiation of periodontal ligament cells on various guided tissue regeneration \\ membranes. J Periodont Res 2001; 36: 322-327. (c) Munksgaard, 2001.
}

The purpose of this study was to evaluate the biological effects of guided tissue regeneration (GTR) membrane materials, per se, on the periodontal tissue regeneration. Rat periodontal ligament (PDL)-derived cells were used to study the attachment, proliferation and differentiation, in vitro, on various GTR membranes. Five commercially available membranes-bovine type I collagen (BioMend ${ }^{\mathbb{R}} ; \mathrm{BM}$ ), bovine type I atelocollagen (Tissue Guide ${ }^{\mathbb{R}} ; \mathrm{TG}$ ), polylactic acid (Epi-Guide ${ }^{\mathbb{R}} ; \mathrm{EG}$ ), co-polymer of polylactic acid and polyglycolic acid (Resolute $\left.{ }^{\mathbb{R}} ; \mathrm{RL}\right)$ and expanded polytetrafluoroethylene: e-PTFE (Gore Tex ${ }^{\circledR}$; GT)-were examined. A $3 \times 3 \mathrm{~mm}$ section of the membrane was fixed to the bottom of a $35 \times 10 \mathrm{~mm}$ style culture dish and plated with $2 \mathrm{ml}$ of cell suspension at an initial density of $5 \times 10^{4}$ cells $/ \mathrm{ml}$ in culture medium with $10 \%$ fetal bovine serum. For cell growth analysis, the specimens were fixed with $10 \%$ buffered formalin and stained with hematoxylin at 1.5 hours and 1, 3 and 5 days after cell seeding. The number of cells included in a unit area of $0.25 \mathrm{~mm}^{2}$ were counted under light microscopy. As a comparative scaffold of cell proliferation, a plastic cover for cell culture slip (Celldesk ${ }^{\mathbb{R}}$ : CD) was used. For analysis of cell differentiation, activity of alkaline phosphatase (ALP) and calcification were histochemically revealed after 2 -week cultivation. The initial number of PDL cells attached to the membrane at 1.5 hours after cell seeding was different among membranes. RL, TG and EG had the same level of attached cell numbers as that on $\mathrm{CD}$, while the cell numbers on GT and BM were significantly lower than that on $\mathrm{CD}(p<0.01)$. The rate of cell proliferation with time also differed among the membranes examined. RL and BM demonstrated a significantly higher number of cells at 5 days than at 1.5 hours $(p<0.01)$. TG had increased numbers of cells at 3 and 5 days after cell seeding. However, there was no statistical difference between the cell numbers at 1.5 hours and 5 days after cell seeding $(p>0.1)$. EG had a similar number of cell attachments to that at 1.5 hours throughout the experimental period. There was almost no cell proliferation on GT. Cell clusters of ALP positive cells and foci of calcification were seen on all membranes except for GT, where a scant number of cells were seen. Results from this study implied that GTR membrane materials, per se, may influence cell proliferation and differentiation in the process of periodontal tissue regeneration.
Takashi Takata ${ }^{1}$, Hom-Lay Wang ${ }^{2}$, Mutsumi Miyauchi ${ }^{1}$

${ }^{1}$ Department of Oral Pathology, Hiroshima University Faculty of Dentistry, Hiroshima, Japan, ${ }^{2}$ Department of Periodontics/ Prevention/Geriatrics, School of Dentistry, The University of Michigan, Ann Arbor, MI, USA
Takashi Takata, D.D.S., Ph.D., Department of Oral Pathology, Hiroshima University Faculty of Dentistry, 1-2-3 Kasumi, Minami-ku, Hiroshima, Japan 734-8553

Tel: +81 822575632

Fax: +81822575619

e-mail: ttakata@ hiroshima-u.ac.jp

Key words: guided tissue regeneration membranes; periodontal ligament cells; proliferation; differentiation

Accepted for publication October 12, 2000
Guided tissue regeneration (GTR) has recently become an essential part of clinical periodontics with its ultimate goal of regenerating lost periodontal structure. A variety of synthetic and naturally derived GTR barriers have been used to facilitate periodontal tissue regeneration (1-4). These barriers may differ in composition and structure, but they all function as mechanical barriers to prevent 
epithelial and connective tissue cell migration to facilitate regenerative potential cells (such as periodontal ligament cells, bone cells, and cementoblasts) to proliferate and migrate into the protected wound area $(5,6)$. However, questions concerning how these barriers, per se, affect the cells around the periodontium remain unanswered, since only limited studies have been reported $(7,8)$. Salonen $\&$ Persson reported in an in vitro model, low protein binding capacity of the expanded polytetrafluoroethylene (e-PTFE, Gore-Tex) material and the rough-textured surface of the e-PTFE inhibited epithelial cell migration (9). Payne et al. further demonstrated that calcium sulfate appeared to facilitate human gingival fibroblast attachment and spreading, whereas cells on e-PTFE and polylactic acid barrier (Guidor) exhibited a morphology not conducive to migration (7). In addition, Gabriel et al. indicated that only a small amount $\left(<4.6\right.$ cells $\left./ \mathrm{mm}^{2}\right)$ of human gingival fibroblasts attached to the e-PTFE, polylactic acid barrier and non-expanded high density PTFE membranes, and that there were no significant differences among membranes (8).

Ideally, barriers should facilitate cell attachment and coronal migration of the progenitor cells from periodontium, since anchorage dependent cells need to adhere to a substrata to be viable in promoting functions such as proliferation, migration, differentiation, and maturation (10). As Machtei et al. indicated, the presence of connective tissue cells on the inner surface of the retrieved membrane is one of the factors that promote periodontal tissue regeneration (11). Initial cell attachment to the barrier may help clot formation and wound stabilization (12). This earlier cell attachment can also act as a barrier stabilizer to minimize membrane micromovement and prevent the future disruption of new attachment formation. So far, limited information is available regarding how these barriers react to periodontal ligament (PDL) cells. Therefore, the purpose of this investigation was to examine the biological effects of various commercially available GTR barrier materials, per se, on the proliferation and differentiation of PDL cells.

\section{Material and methods}

\section{Cell cultures}

PDL cells were obtained from extracted molar teeth of 8-week-old Lewis male rats. Molar teeth were put on a culture dish and outgrowth from the PDL attached on the molar roots was subcultured in Dulbecco's Modified Eagle Medium with 10\% fetal bovine serum (FBS). The cells less than passage 5 were used for the analyses described below.

\section{Membranes examined}

Five commercially available membranes with different composition and structures were examined; a bovine type I collagen membrane (BioMend ${ }^{\mathbb{R}}$; BM, CALCITEK, CA, USA), a bovine type I atelocollagen membrane (Tissue Guide ${ }^{\mathbb{R}}$; TG, KOKEN, Tokyo, Japan), a co-polymer of polylactic acid and polyglycolic acid membrane (Resolute ${ }^{\circledR} ;$ RL, W.L. Gore \& Associates, AZ, USA), a polylactic acid membrane (Epi-Guide ${ }^{\mathbb{R}}$; EG, THM Biomedical Inc., MN, USA) and an expanded polytetrafluoroethylene (e-PTFE) membrane (Gore Tex ${ }^{\circledR}$; GT, W.L. Gore \& Associates, AZ, USA).

\section{Proliferation assay}

A $3 \times 3 \mathrm{~mm}$ section of the membrane was fixed to the bottom of a $35 \times 10 \mathrm{~mm}$ style culture dish with a double-faced adhesive tape and plated with $2 \mathrm{ml}$ of cell suspension at an initial density of $5 \times 10^{4}$ cells $/ \mathrm{ml}$ in culture medium with $10 \%$ FBS. The specimens were fixed with $10 \%$ buffered formalin and stained with hematoxylin at 1.5 hours and 1, 3 and 5 days after cell seeding. The observation periods were determined by preliminary experiments where the proliferation assay was tried for 7 days more. From 7 days on, cells became very flat and counting of the cell number was difficult. The number of cells included in a unit area of $0.25 \mathrm{~mm}^{2}$ in 5 randomly selected areas on each sample was counted under a magnification of $\times 400$, using a light microscopy equipped with an ocularmicrometer. As a comparative scaffold of cell proliferation, a plastic cover for cell culture slip $\left(\right.$ Celldesk ${ }^{\circledR}$ : CD, Sumitomo Bakelite, Tokyo, Japan) was prepared for the assay in the same procedures. All samples were, at least, triplicated. A total of 15 areas from triplicated samples for each membrane were counted and converted into the mean cell number per $\mathrm{mm}^{2} \pm$ standard error.

\section{Histochemical analyses of alkaline phosphatase (ALP) activity and mineralization}

For analysis of cell differentiation on the membranes, activity of ALP and calcification were histochemically revealed after 2-week cultivation in the above-mentioned culture medium, with $50 \mu \mathrm{g} / \mathrm{ml}$ ascorbic acid, $10 \mathrm{~mm} \beta$-glycerophosphate and $10^{-8} \mathrm{M}$ dexamethasone. ALPase activity was histochemically demonstrated with naphtol AS-BI phosphate as substrate and new fuchsin and sodium nitrate as couplers. Mineralization was revealed by alizarin red $\mathrm{S}$ staining. 


\section{Statistical analysis}

The results were statistically analyzed using Wilcoxon's test for non-paired examination. The significance level for rejection was chosen at $p<0.05$.

\section{Results}

\section{Cell proliferation on the membranes}

During the experimental period, there was no evidence indicating any influence from membrane toxic residues or from the glue from the doublesided adhesive tape. PDL cell growth on a culture dish adjacent to the membranes and tape was not affected by the membranes or the glue.

PDL cells were seen as rounded cells on the membranes at 1.5 hours after cell seeding (Fig. 1). The initial number of PDL cells attached to the membrane at 1.5 hours after cell seeding was different among the membranes (Figs. 1 and 2). RL, TG and EG showed around the same level of attached cell numbers as that on $\mathrm{CD}$, while the cell numbers on GT and BM were significantly lower than that on $\mathrm{CD}(p<0.01)$.

PDL cells seeded on CD demonstrated linear proliferation with time after a 24-hour quiescence period (Fig. 2). The rate of cell proliferation with time was different among the membranes examined (Fig. 2). PDL cells became spindle or stellate shape and proliferated along the fibrous or porous structures of the membranes (Fig. 1). RL and BM had significantly higher numbers of cells at 5 days after cell seeding than the initial number of cell attachments at 1.5 hours $(p<0.01)$. TG also showed increased number of cells at 3 and 5 days after cell seeding compared to the initial number of attached cells (Fig. 2). However, the cell growth on TG was kept less than double in number throughout the experimental period and there was no statistical difference between the cell numbers at 1.5 hours and 5 days after cell seeding $(p>0.1)$. Although the cell growth on RL and TG reached a stationary phase 3 days after cell seeding, BM showed linear growth of PDL cells throughout the experimental period (Fig. 2). During the experimental period, the number of cells on EG was maintained at almost the same level as that at 1.5 hours (Fig. 2). There was almost no cell proliferation on GT (Figs. 1 and 2).

\section{Alkaline phosphatase activity and mineralization of the cells on the membranes}

PDL cells cultured on a plastic plate showed distinct activity of ALP and formed mineralized

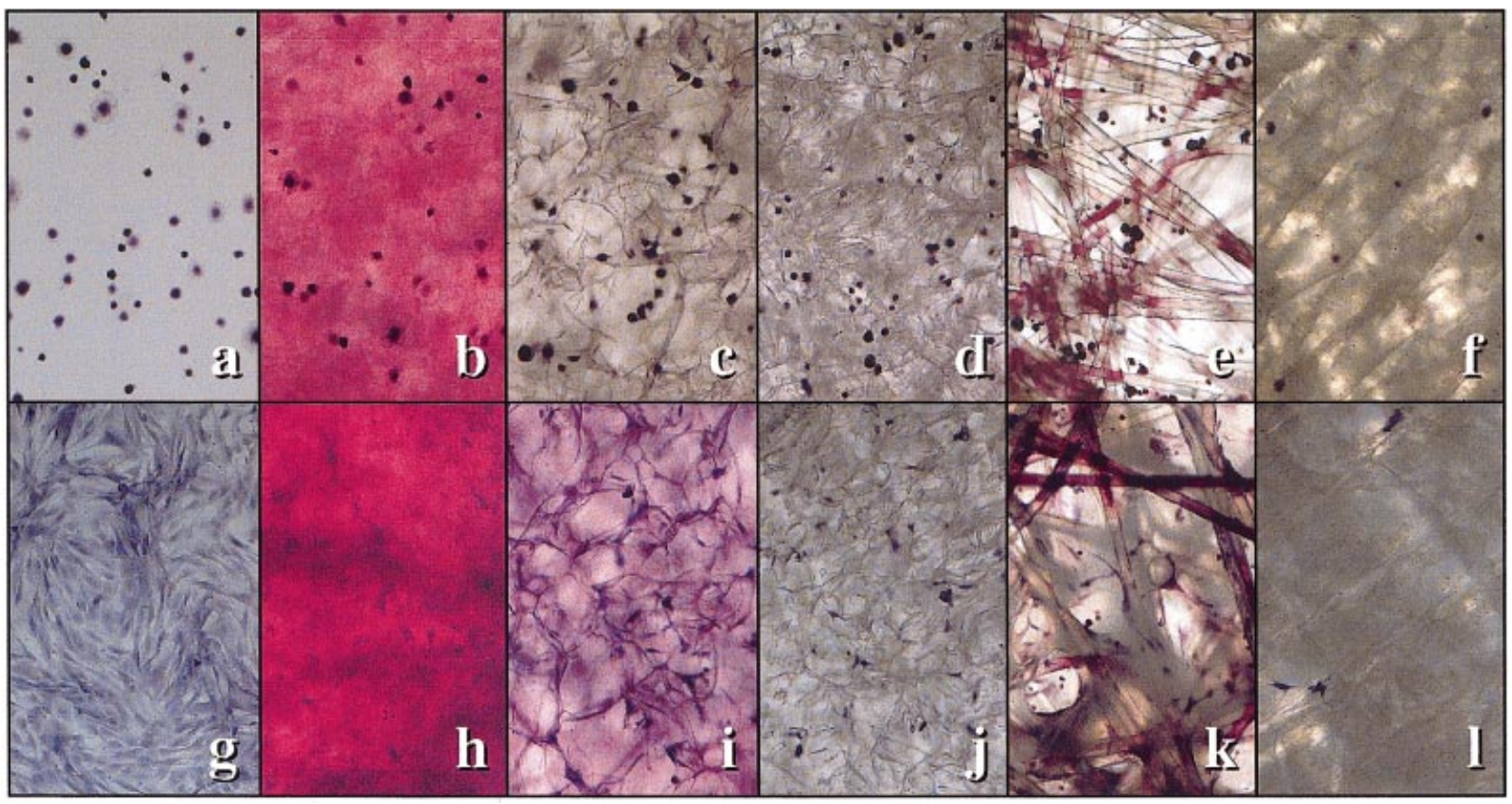

Fig. 1. Periodontal ligament (PDL) cells on various membranes at 1.5 hours (top) and 5 days (bottom). a \& g, a plastic cover for cell culture slip (CD), b \& h, a bovine type I collagen membrane (BM), c \& i, a bovine type I atelocollagen membrane (TG), d \& j, a co-polymer of polylactic acid and polyglycolic acid membrane (RL), e \& k, a polylactic acid membrane (EG) and f \& 1, an e-PTFE membrane (GT). PDL cells were seen as rounded cells on the membranes at 1.5 hours after cell seeding (top). The initial number of PDL cells attached to the membrane was different among membranes. PDL cells were spindle or stellate shape and proliferated along the fibrous or porous structures of the membranes at day 5 (bottom). The rate of cell proliferation with time was different among the membrane examined. Hematoxylin staining $\times 50$. 
foci after 2-week cultivation in the culture medium supplemented with the mineralization enhancers (Fig. 3). PDL cells on the tested membranes, except for GT where only a scant number of cells was seen, showed ALP activity (Fig. 3). Foci of calcification

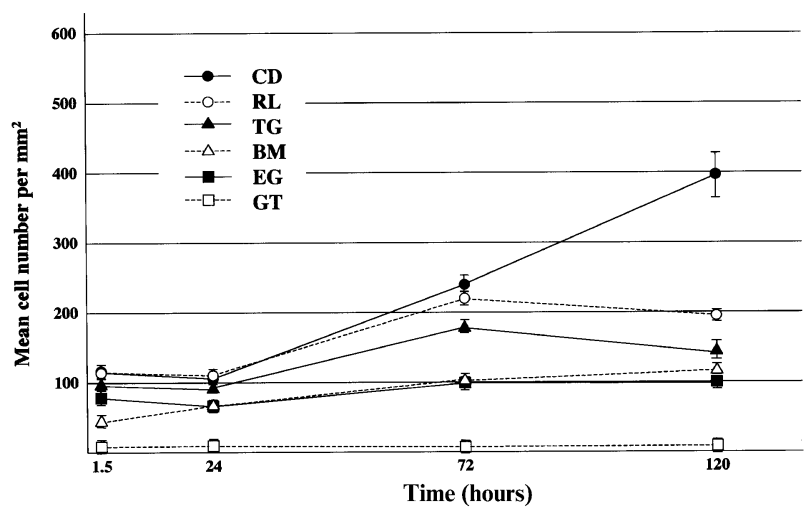

Fig. 2. Number of periodontal ligament cells on various membranes examined. $\mathrm{CD}=$ plastic cover for cell culture slip; $\mathrm{RL}=\mathrm{a}$ co-ploymer of polylactic acid and polyglycolic acid membrane; $\mathrm{TG}=$ a bovine type $\mathrm{I}$ atelocollagen membrane; $\mathrm{BM}=\mathrm{a}$ bovine type I collagen membrane; $\mathrm{EG}=$ a polylactic and membrane; $\mathrm{GT}=$ an e-PTF membrane were also noted on BM, EG and RL. TG was strongly stained with alizarin red $\mathrm{S}$ and mineralization foci were not clearly distinguished. There was no mineralization on GT (data not shown).

\section{Discussion}

It has been demonstrated that the theory of GTR (i.e. selective repopulation of the root surface by multipotential cells) is a viable, but not a predictable treatment option through the utilization of the various membranes. Barrier membranes for GTR therapy must meet certain requirements such as tissue integration to promote wound stabilization, cell occlusivity to attract desirable cells, space-making and/or maintaining to allow new attachment formation, and biocompatibility to ensure material safety (13). Other factors such as clinical manageability and reasonable cost should also be considered. Among these preferred requirements, the biocompatibility is an important criterion for determining which device to choose. Toxicity, pyrogenic and hemolytic activity, and antigenicity must also be extensively examined under in vitro and in vivo conditions prior to

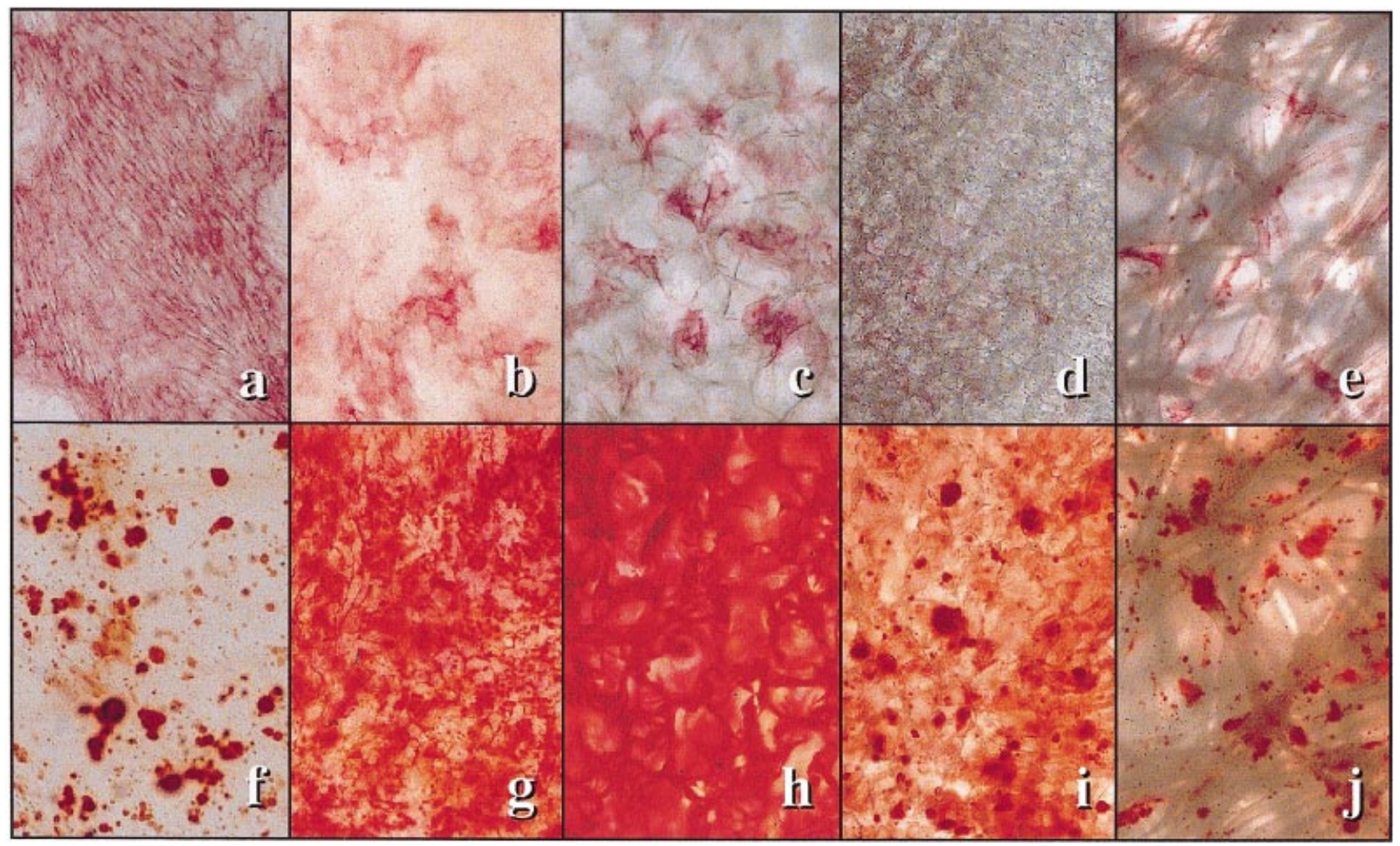

Fig. 3. Alkaline phosphatase activity of periodontal ligament cells (top) and mineralization (bottom). a \& f, a plastic cover for cell culture slip (CD), b \& g, a bovine type I collagen membrane (BM), c \& h, a bovine type I atelocollagen membrane (TG), d \& i, a co-polymer of polylactic acid and polyglycolic acid membrane (RL), e \& j, a polylactic acid membrane (EG). Distinct activity of ALP and formation of mineralized foci were seen not only on CD but also on all tested membranes except for GT (an e-PTFE membrane), where only a scant number of cells was seen (data not shown). It is difficult to distinguish mineralization foci on TG, which is strongly stained with alizarin red $\mathrm{S}(\mathrm{h}) . \times 60$. 
clinical usage. Furthermore, the membranes should also possess the ability to enhance cell attachment, proliferation and differentiation. Various barriers have been developed to meet these requirements. However, factors such as membrane constituents, morphology, surface textures, size of perforations and duration of barrier function that may influence GTR outcomes have not yet been completely understood. The data from this study demonstrate PDL cell attachment, proliferation and differentiation when exposed to different GTR barrier membranes.

Under the conditions of the present in vitro experiments, attachment of PDL cells on GTR membranes was different among various membranes examined. Among the membranes tested, RL, TG and EG illustrated a good level of initial cell attachment, similar to that on CD. BM showed significantly less initial attachment of cells, while GT had only a minimal level of initial cell attachment. This suggests that the growth rate of PDL cells was influenced by the material used. It may imply that barrier materials' constituents, surface roughness and certain other properties, per se, influence cell proliferation during GTR therapy. This supports the concept proposed by Burridge and his coworkers, that inherent to cellular migration over a substrate (barrier) is the ability of cells to first attach to the substrate surface (14). Attachment of cells to substrates involves a four-step sequence that includes adsorption of glycoproteins to the substrate surface, cell contact, attachment, and spreading (14). Generally, cell migration and proliferation begin only after these events have occurred. In addition, results from this study indicated that all barriers tested are biocompatible and non-toxic.

Data obtained from this study indicated that some of GTR membranes examined here seem to provide a favorable scaffold for PDL cells to proliferate and migrate. RL, TG and BM showed cell proliferation but the cell growth on RL and TG reached a stationary phase 3 days after cell seeding. The number of cells on EG was maintained at the same level as at initial attachment. No cell proliferation on GT was noted, while CD showed linear proliferation of PDL cells with time. Although the cell number on $\mathrm{CD}$ at 5 days after cell seeding was around 4 times the initial number of cell attachments, cell growth was kept less than double in number throughout the experimental period on the tested membranes, except for BM. BM demonstrated linear cell growth and it increased to more than double at 5 days after cell seeding.

$\mathrm{RL}$ is composed of a porous structure of glycolide polymer fiber and an occlusive membrane of glycolide and lactide copolymer. EG is a hydrophilic membrane formed from polylactic acid with a flexible open cell structure. These porous or open cell structures of the polymer membranes were designed for uptake of fluid blood that facilitates adherence to the tooth surface in vivo. In addition, the internal void spaces of the membranes support a forming blood clot and influence the invasion of cells into its matrix. Results obtained from this study demonstrated that RL had a higher PDL cell attachment and proliferation than EG. It was an expected finding that cells attached on the fibers and migrated on them. PDL cells seemed to have a good affinity to the glycolide polymer. However, Simain-Sato et al. reported that fibroblasts cultured on RL showed rounded oval cells and cell fragments (15). A similar finding was also illustrated by Payne et al. (7) The difference noted in these results may be due to the use of two different cell types-Simain-Sato et al. and Payne et al. used gingival fibroblasts (from rat and human), whereas PDL ligament cells from rat were used in the present study. Further study using elimination by substitution is needed to clarify which specific factor has more effect on cell proliferation.

Although BM and TG were both belonging to collagen devices, initial cell attachment and subsequent cell proliferation were different on these membranes. Linear proliferation of PDL cells with time was observed on BG, but the cell growth on TG reached a stationary phase 3 days after cell seeding and was kept less than double in number throughout the experimental period. BM is a membrane consisting of cross-linked fibrillar collagen from tendon and TG is mainly composed of cross-linked amorphous atelocollagen. The differences in cross-linkage of collagen fibrils, surface roughness and structure of collagen molecules may account for the different effects on PDL cell attachment and proliferation. However, the result observed here is in agreement with Locci et al., who found that extracellular matrix, which consists primarily of collagen and chondroitin4-sulphate, was the most suitable device to stimulate both cellular proliferation and extracellular macromolecule accumulation (16). This implies that collagen membranes may promote formation of a thin osteoblastic cell layer to eventually enhance bone regeneration. However, further study is certainly needed to confirm these hypotheses.

Our data indicated that GT had only a minimal cell attachment and proliferation. This is in agreement with Salonen \& Persson (9). They examined epithelial cell migration on three different devices; hydroxyapatite, teflon and GT. Data from this study showed that hydroxyapatite had the most cells attached, while teflon and GT had almost no cell attachment. Winter (17) also suggested that GT microstructure tends to retard cell attachment and migration across the 
membrane by contact inhibition. Similar findings have also been reported elsewhere $(6,15,16)$. The lack of adherence may be due to the decreased wettability, the surface roughness created by the overlapping fibrils and/or the low protein binding capacity. The minimal tissue integration to GT may be an advantage for membrane retrieval. However, this may also create potential problems for initial clot formation, wound stabilization and membrane stability and thus may interfere with wound healing. Hence, the advantages of minimal cell attachment of GT during GTR remained to be explored.

Differentiation of PDL cells to mineralizing cells (e.g. cementoblastic and/or osteoblastic cells) was monitored by histochemical staining of alkaline phosphatase and mineralization foci. Although the rates of proliferation of PDL cells were different among membranes, distinct positive staining for alkaline phosphatase and calcification was seen on all membranes examined except for GT. The alkaline phosphatase activity and mineralization of PDL cells could not be assessed on the GT barrier, since there was only a few cells attached. The results indicated that the membranes do not impede cellular differentiation.

One must be cautious in interpreting results obtained by using an in vitro experimental model, since it cannot recreate the complex interactions of cells in vivo. Other limitations in this study include (but are not limited to a short study period (i.e. 3-5 days) and a single cell line (i.e. PDL cells). Future studies should include a longer follow-up period as well as testing other cells (e.g. epithelial cells, connective tissue fibroblasts, PDL cells, bone cells, cementoblasts and other unidentified cells) that are involved in the GTR process.

Within the limit of this study, it is concluded that GTR membrane materials, per se, may influence periodontal ligament cell proliferation and differentiation in the process of periodontal tissue/bone regeneration. Among the five membranes examined, although none of them showed active cell growth as seen on $\mathrm{CD}, \mathrm{BM}$ showed linear proliferation of PDL cells with time and a favorable scaffold for differentiation. Further predictable success of GTR can be enhanced as a result of understanding the biology of tissues surrounding these devices and knowing the physical and chemical structure of the materials used.

\section{Acknowledgements}

This study was supported in part by grants-in-aid No.10557159 from the Ministry of Education, Science and Culture of Japan and the University of Michigan, Periodontal Graduate Student Research Fund.

\section{References}

1. Wang HL, O’Neal RB, Thomas CL, Shyr Y, MacNeil RL. Evaluation of an absorbable collagen membrane in treating Class II furcation defects. J Periodontol 1994; 65:1029-1036.

2. Yukna CN, Yukna RA. Multi-center evaluation of bioabsorbable collagen membrane for guided tissue regeneration in human Class II furcations. J Periodontol 1996; 67:650-657.

3. Becker W, Becker BE, Mellonig J et al. A prospective multicenter study evaluating periodontal regeneration for Class II furcation invasions and intrabony defects after treatment with a bioabsorbable barrier membrane: 1-year results. J Periodontol 1996;67:641-649.

4. Cortellini P, Pini Prato G, Tonetti MS. Periodontal regeneration of human infrabony defects. I. Clinical measures. J Periodontol 1993;64:254-260.

5. Nyman S, Gottlow J, Karring T, Lindhe J. The regenerative potential of the periodontal ligament. An experimental study in the monkey. J Clin Periodontol 1982; 9:257-265.

6. Melcher AH. On the repair potential of periodontal tissues. J Periodontol 1976;47:256-260.

7. Payne JM, Cobb CM, Rapley JW, Killoy WJ, Spencer P. Migration of human gingival fibroblasts over guided tissue regeneration barrier materials. J Periodontol 1996; 67:236-244.

8. Gabriel BF, Rivera-Hidalgo F, Stanford TW, Dill RE. Attachment of fibroblasts to membranes used for guided tissue regeneration in vitro. J Dent Res 1996;75:326.

9. Salonen JI, Persson GR. Migration of epithelial cells on materials used in guided tissue regeneration. $J$ Periodont Res 1990;25:215-221.

10. Grinnell F. Cellular adhesiveness and extracellular substrata. Int Rev Cytol 1978;53:65-144.

11. Machtei EE, Cho MI, Dunford R, Norderyd J, Zambon JJ, Genco RJ. Clinical, microbiological, and histological factors which influence the success of regenerative periodontal therapy. J Periodontol 1994;65:154-161.

12. Wikesjo UM, Sigurdsson TJ, Lee MB, Tatakis DN, Selvig KA. Dynamics of wound healing in periodontal regenerative therapy. J Calif Dent Assoc 1995;23:30-35.

13. Scantlebury TV. 1982-1992: a decade of technology development for guided tissue regeneration. $J$ Periodontol 1993;64:1129-1137.

14. Burridge K, Molony L, Kelly T. Adhesion plaques: sites of transmembrane interaction between the extracellular matrix and the actin cytoskeleton. $J$ Cell Sci Suppl 1987;8: 211-229.

15. Simain-Sato F, Lahmouzi J, Kalykakis GK, et al. Culture of gingival fibroblasts on bioabsorbable regenerative materials in vitro. J Periodontol 1999;70: 1234-1239.

16. Locci P, Calvitti M, Belcastro S et al. Phenotype expression of gingival fibroblasts cultured on membranes used in guided tissue regeneration. J Periodontol 1997; 68:857-863.

17. Winter GD. Transcutaneous implants: reactions of the skin-implant interface. J Biomed Mater Res 1974; 8:99-113. 